\title{
Pulmonary manifestation of systemic mast cell disease
}

\author{
M. Schmidt*, C. Dercken*, O. Loke*, S. Reimann", S. Diederich ${ }^{+}$, S. Blasius ${ }^{\S}$, S. Heidenreich*
}

\begin{abstract}
Pulmonary manifestation of systemic mast cell disease. M. Schmidt, C. Dercken, O. Loke, S. Reimann, S. Diederich, S. Blasius, S. Heidenreich. C) ERS Journals Ltd 2000.

ABSTRACT: Systemic mast cell disease is a rare disease of unknown aetiology. Systemic infiltration and proliferation of mast cells in skin, bone marrow, gastrointestinum and lymph nodes is the central pathological feature. This study reports a patient with mastocytosis of the skin (urticaria pigmentosa) for 10 yrs. The patient was referred to hospital for dyspnoea. Chest radiograph showed moderate reticular infiltration of both lungs, computerized tomography revealed multiple lymph nodes of the mediastinum and faint nodular lesions of middle and upper areas of lungs. Transbronchial biopsy demonstrated mast cell infiltration of the lung with formation of mast cell granuloma. According to the current literature, systemic mast cell disease with pulmonary involvement is a very rare entity. After a treatment with interferon $\alpha$ 2a over 6 months, the patient's condition and particularly dyspnoea showed improvement in parallel with an amelioration of the lesions as demonstrated by thorax computed tomography.
\end{abstract}

Eur Respir J 2000; 15: 623-625.
Depts of *Medicine, ${ }^{\#}$ Dermatology, and ${ }^{+}$Radiology, and ${ }^{\S}$ Gerhard Domagk Institute of Pathology, University of Münster, Münster, Germany.

Correspondence: S. Heidenreich, Dept of Medicine D, University Hospital Münster, Albert-Schweitzer-Str. 33, D-48129 Münster, Germany, Fax: 492518347545.

Keywords: Interferon

lung infiltration

mast cell

mastocytosis

urticaria

Received: November 271998

Accepted after revision July 21999
Mastocytosis is a local or systemic disease characterized by infiltration and expansion of mast cells in various tissues. Urticaria pigmentosa is diagnosed when skin is affected systemically showing multiple brown or livid papules.

Whenever mast cell infiltration involves additional organs, the diagnosis of systemic mast cell disease (SMCD) is applied. Involvement of the gastrointestinal tract and bone marrow is most common. The patient presented here showed systemic mastocytosis with a biopsy-proven mast cell infiltration of the bone marrow and lung.

\section{Case report}

The present 54-yr-old male with a moderate smoking history had chronic pruritus for 10 yrs. Integument revealed exanthematic red brown papules with major involvement of the truncus. Rubbing of the skin evoked urticarial swelling. A cutaneous skin biopsy showed keratinocytes with increased pigmentation and proliferation of melanocytes. In the upper dermis, perivascular infiltrates consisted of lymphocytes, histiocytes and huge numbers of mast cells, all alterations indicative for urticaria pigmentosa. A total of 24 mast cells per high power field (magnification $\times 400$ ) were counted. Figure 1 shows the truncal skin of the patient with the typical dermal manifestation of mast cell disease. The patient then presented with dyspnoea. The chest radiograph showed signs of interstitial lung disease with a fine reticular infiltration of both lungs (fig. 2A). Computed tomography (CT) of the thorax revealed faint nodular and cystic lesions of the middle and upper fields of the lungs with mediastinal adenopathy (fig. 2B). Blood gas analysis at rest gave a mild hypoxaemia with an arterial oxygentension $\left(\mathrm{Pa}_{\mathrm{a}} \mathrm{O}_{2}\right)$ of $8.5 \mathrm{kPa}(64 \mathrm{mmHg})$. Pulmonary function tests showed a modest obstruction with a forced expiratory volume in one second (FEV1) of $3.05 \mathrm{~L}$ ( $77 \%$ of predicted value)

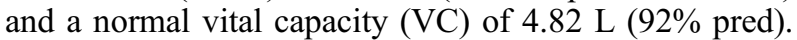
Carbon monoxide transfer factor $(T \mathrm{~L}, \mathrm{CO})$ was markedly diminished $(55 \%$ pred). Laboratory chemical examinations yielded a peripheral white blood count of 12.250 cells $\cdot \mathrm{mm}^{-3}$ with $65 \%$ neutrophiles, $25 \%$ lymphocytes, $5 \%$ monocytes, 4\% eosinophilic cells and 1\% basophilic cells. Red blood counts and thrombocytes were normal. Standard laboratory values of serum were all in normal range. Serum tryptase was $2.86 \mu \mathrm{g} \cdot \mathrm{mL}^{-1}$ (normal range $<1 \mu \mathrm{g} \cdot \mathrm{mL}^{-1}$ ), histamine in $24 \mathrm{~h}$ urine collection was 72 $\mu \mathrm{g} \cdot 24 \mathrm{~h}^{-1}$ (normal $<50 \mu \mathrm{g} \cdot 24 \mathrm{~h}^{-1}$ ).

Biopsy of the bone marrow was performed and showed typical mast cell granuloma with focal cellular accumulations. Bone marrow presented a mast cell infiltration of $2 \%$, lobulated mast cell nuclei were not found and fat cell content was normal. Because of the clinical symptoms

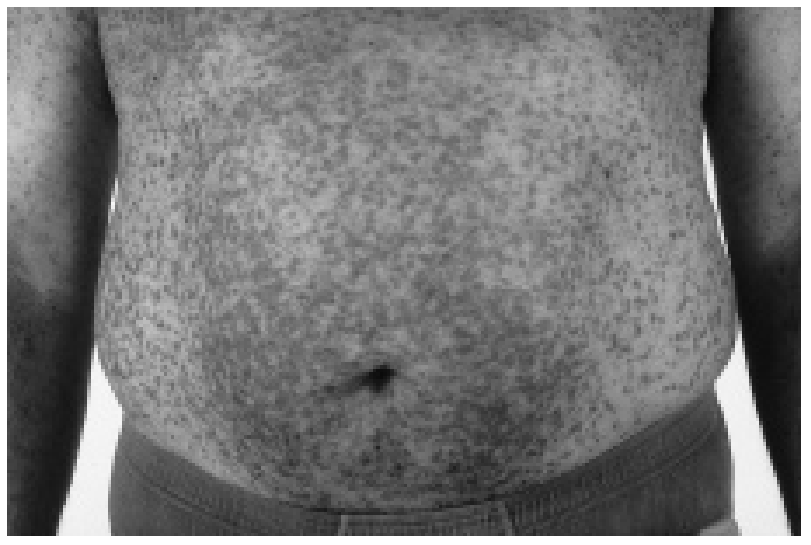

Fig. 1. - Torso of the presented patient showing generalized papulosis. After rubbing, urticarial skin lesions develop indicative for urticaria pigmentosa. 

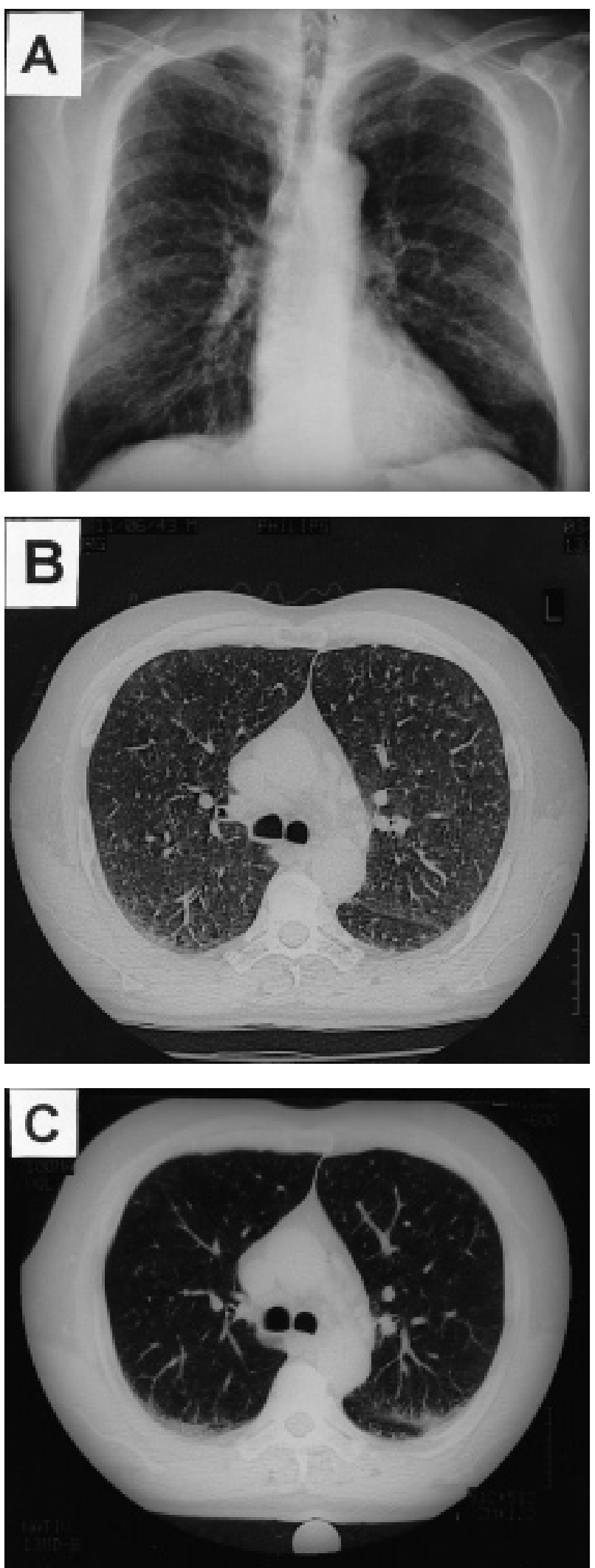

Fig. 2. - Chest radiograph (A) showing diffuse fine reticular interstitial infiltration pattern of both lungs. Computed tomography (CT) scan of the thorax shows faint cystic and nodular lesions of lung interstitium as well as adenopathy of the mediastinum at the time before interferon- $\alpha$ treatment (B). After 6 months of treatment with interferon- $\alpha$ interstitial lesions of the lung had markedly improved (C). of the patient and the described radiological findings, bronchoscopy and transbronchial fine needle biopsy was carried out. Bronchoscopy revealed signs of chronic bronchitis.

Histology showed infiltration of the lung interstitium by mast cells (basophilic cytoplasma and granula) with abortive mast cell granuloma formation. As shown in figure 3, mast cell granuloma consisted of lung interstitial cells, lymphocytes and mast cells. Mast cells showed signs of maturity as well as of immaturity which were distinguishable by the content of mast cell granula and size and shape of nuclei. A significant fibrosis was not visible.

Gastroscopy showed no pathological condition and biopsies could not demonstrate mast cell infiltration of the gastrointestinum. Cardiac evaluation including echocardiography was normal. For urticaria pigmentosa, therapy with antihistaminic agents was continued, and his pulmonary symptoms inhalative mast cell stabilizers (disodium chromoglycate) were applied. Since dyspnoea did not improve after 6 weeks, therapy with interferon $\alpha-2 \mathrm{a}$ (Roferon $(\mathrm{B}$; Roche, Grenzach, Germany) was started at a dose of 3 million IU. IE Roferon s.c. three times a week. After 6 months of treatment, CT of the thorax showed improvement of nodular and cystic lesions of the lung (fig. 2C). Size and number of mediastinal lymph nodes did not decrease significantly and pulmonary function tests were stable.

\section{Discussion}

Mastocytosis represents a wide spectrum of disorders characterized by proliferation and accumulation of tissue mast cells. In most patients, mast cell expansion is confined to the skin giving the diagnosis of cutaneous mastocytosis. In systemic mastocytosis different organs can be involved with the bone marrow and the gastrointestinal tract as the most frequently affected sites [1-3].

The lung is typically not involved in systemic mastocytosis although some studies described pulmonary involvement $[4,5]$. In a very early study, solitary pulmonary mast cell granuloma or tumours have been described but at the time, pathologists had difficulty in distinguishing

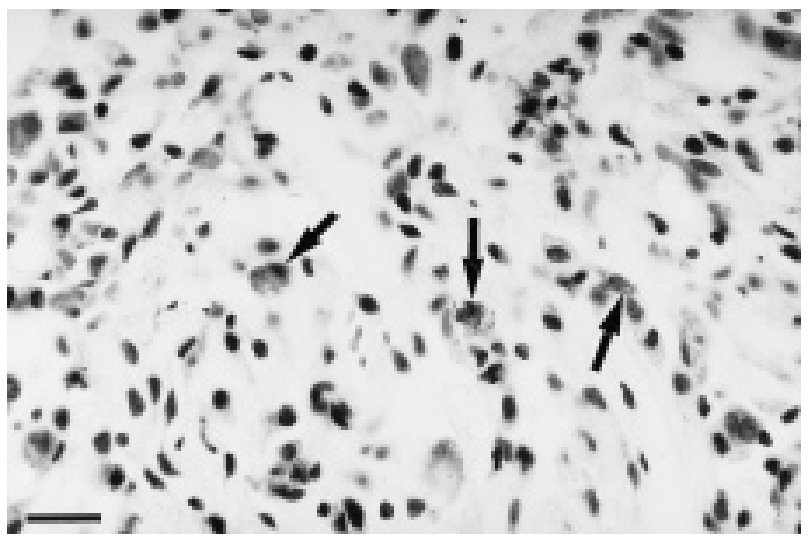

Fig. 3. - Abortive mast cell granuloma formation of lung interstitium. The section shows interstitial infiltration by lymphocytes and mast cells besides resident lung interstitial and alveolar cells. Mast cells are characterized by deep violet-blue basopholic granules and cytoplasm (arrows). Mature as well as immature mast cells with different shape of nuclei and content of granules are distinguishable. (Giemsa stain; original magnification $\times 240$, internal scale bar $=50 \mu \mathrm{m}$.) 
the histological pattern of mast cell tumours from plasmacytoma because staining procedures and immunohistochemical markers were not well-established [6].

According to a study by TRAVIS et al. [7], where 58 patients with systemic mastocytosis were evaluated, respiratory symptoms occurred in $20 \%$ of the SMCD patients. The symptoms were dyspnoea and chronic bronchitis sometimes in association with asthma. Chest radiographic abnormalities were found in $16 \%$ of the patients. The abnormalities described were focal or scattered areas of fibrosis, bilateral interstitial fibrosis and multiple pulmonary nodules. In this study, suspected pulmonary involvement by mastocytosis was not confirmed by histology.

Concerning therapy of SMCD, all modes of treatment are aimed to relieve symptoms because curative therapy does not exist. Symptomatic treatment includes H1- and H2-antihistaminic drugs, prostaglandin blockers and mast cell stabilizers such as oral disodium cromoglycate [8-10].

For systemic and malignant mastocytosis different chemotherapeutical schemes exist all of which have been shown to be of limited value. Treatment with prednisolone and interferon $\alpha$-2a has failed to improve the natural course of the disease but some cases showing a histologically proven response and relief of symptoms have been published [11-13]. Progression of symptoms under conventional therapy was the rationale to treat the presented patient with interferon $\alpha-2 \mathrm{a}$. After treatment with this drug for 6 months, pulmonary function tests, the number of enlarged mediastinal lymph nodes and biochemical markers analysed in serum or urine did not improve significantly but symptoms such as dyspnoea and pruritus improved and computed tomography of the lung showed amelioration of interstitial lesions.

\section{References}

1. Tharp MD. The spectrum of mastocytosis. Am J Med Sci 1985; 289: 119-132.

2. Longley J, Duffy TP, Kohn S. The mast cell and mast cell disease. J Am Acad Dermatol 1995; 32: 545-561.

3. Golkar L, Bernhard JD. Mastocytosis. Lancet 1997; 349: 1379-1385.

4. Huang T-Y, Yam LT, Li C-Y. Radiological features of systemic mast-cell disease. Br J Radiol 1987; 60: 765770 .

5. Avila NA, Worobec AS, Ling A, Hijazi Y, Metcalfe D. Pulmonary and ovarian manifestations of systemic mastocytosis. Am R Ray Soc 1996; 166: 969-970.

6. Charrette EE, Mariano AV, Laforet EG. Solitary mast cell "tumour" of lung. Arch Intern Med 1966; 118: 358-362.

7. Travis WD, Li CY, Bergstrahl EJ, Yam LT, Swee RG. Systemic mast cell disease. Medicine 1988; 67: 345-368.

8. Metcalfe DD. The treatment of mastocytosis: an overview. J Invest Dermatol 1991; 96: 55S-59S.

9. Gasior-Chrzan B, Falk ES. Systemic mastocytosis treated with histamine H1 and H2 receptor antagonists. Dermatology 1992; 184: 149-152.

10. Soter NA, Austen KF, Wassermann SI. Oral disodium cromoglycate in the treatment of systemic mastocytosis. N Engl J Med 1979; 301: 465-469.

11. Kluin-Nelemans HC, Jansen JH, Breukelman H, Wolthers BG. Response to interferon alfa $2 \mathrm{~b}$ in a patient with systemic mastocytosis. New Engl J Med 1992; 326: 619623.

12. Lippert U, Henz BM. Long-term effect of interferon alpha treatment in mastocytosis. Brit J Dermatol 1996; 134: 1164-1165.

13. Butterfield JH. Response of severe systemic mastocytosis to interferon alpha. Br J Dermatol 1998; 138: 489-495. 